\title{
NOTES ON RENEWAL SYSTEMS
}

\author{
SUSAN WILLIAMS
}

(Communicated by R. Daniel Mauldin)

\begin{abstract}
A renewal system is a symbolic dynamical system generated by free concatenations of a finite set of words. Renewal systems are sofic, but not every sofic shift is topologically conjugate to a renewal system.
\end{abstract}

For background on symbolic dynamical systems and sofic shifts see, for example, [M] or [BMT]. Let $A$ be a finite alphabet. A subshift $R$ of $A^{Z}$ is called a renewal system if there is a finite set $W$ of finite strings (words) over $A$ such that each element of $R$ can be obtained as an infinite bilateral concatenation of elements of $W$. This term is due to Roy Adler, whom I thank for suggesting this area of study. The set $W^{*}$ of finite concatenations of words of $W$ is extensively studied in automata theory, but the dynamical properties of renewal systems are in general not well understood.

It is easy to see that every renewal system is sofic. A subshift $S$ of $A^{Z}$ is sofic if and only if its language (the set of words appearing in elements of $S$ ) is regular [W]; the language of $R$ is the set of subwords of $W^{*}$, which is regular. Alternatively, let the words of $W$ be $a_{1}^{(i)} \ldots a_{l_{i}}^{(i)}, i=1, \ldots, k$ and let $x_{j}^{(i)}, i=$ $1, \ldots, k, j=1, \ldots, l_{i}$ be distinct symbols. The renewal system $x$ generated by the words $x_{1}^{(i)} \ldots x_{l_{i}}^{(i)}, i=1, \ldots, k$ is a shift of finite type of a special sort we call a loop system because of the appearance of the associated directed graph (Figure 1). $R$ is the image of $x$ under the 1-block map $x_{j}^{(i)} \rightarrow a_{j}^{(i)}$ and hence is sofic.

It is also easy to see that not every sofic shift, or even every shift of finite type, is a renewal system. For example, the finite type shift $\Sigma$ given by the directed graph in Figure 2 (here we take the vertices rather than the edges as the alphabet of our shift) is not a renewal system. A generating set would have to contain words of the form $a^{m}, b^{n}$ to produce the sequences $a^{\infty}, b^{\infty}$ (here powers denote concatenation); but the word $b^{n} a^{m}$ may not appear in $\Sigma$. However, $\Sigma$ is topologically conjugate to a renewal system: the 1-block map $a \rightarrow a, b \rightarrow b$,

Received by the editors September 12, 1989.

1980 Mathematics Subject Classification (1985 Revision). Primary 54H20; Secondary 28D05, $68 \mathrm{Q} 45$.

The author gratefully acknowledges the support of the IBM T. J. Watson Research Center and the National Science Foundation (Grant DMS-8704642). 


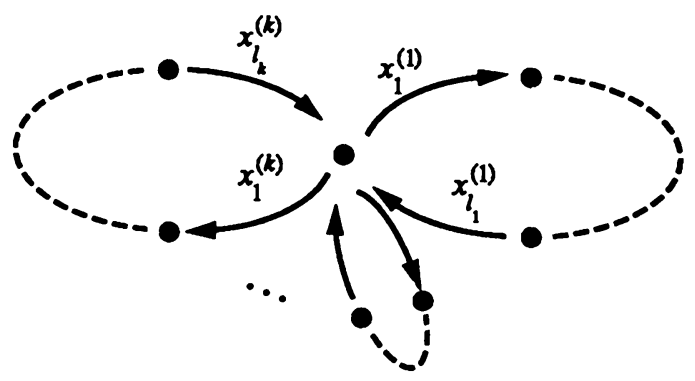

FIGURE 1

$c \rightarrow b$ has a 2-block inverse, and the image of $\Sigma$ is the renewal system generated by $\{a, b b, b b b\}$.

The main result of this paper is that not every sofic shift is topologically conjugate to a renewal system. The example given is not exotic: similar arguments will apply to many sofic shifts. But it is not known if every irreducible shift of finite type is conjugate to a renewal system. (For recent work in this direction see [GLS].)

Example. The sofic shift $S$ given by the labeled directed graph in Figure 3 is not conjugate to a renewal system.

Proof. Suppose $S$ is conjugate to a renewal system $R$. Let the conjugacy $\varphi$ and its inverse be given by block codes with memory and anticipation $n$, which we will also denote by $\varphi$ and $\varphi^{-1}$. Thus $(\varphi(x))_{0}=\varphi\left(x_{-n} \ldots x_{n}\right)$ for $\left(x_{i}\right) \in S$. The image of the fixed point $a^{\infty}$ in $S$ is a fixed point $\hat{a}^{\infty}$ in $R$, so $\varphi\left(a^{m}\right)=\hat{a}^{m-2 n}$ for $m>2 n$, and $\varphi\left(a^{m} b a^{m}\right)$ has the form $\hat{a}^{m-2 n} b_{-n} \ldots$ $b_{n} \hat{a}^{m-2 n}$. Since the point $a^{\infty} b a^{\infty}$ in $S$ is the unique preimage of $\hat{a}^{\infty} b_{-n} \ldots$ $b_{n} \hat{a}^{\infty}$ (where for definiteness we may take these points to have zero coordinate $b, b_{0}$ respectively), we must have $\varphi^{-1}\left(\hat{a}^{k} b_{-n} \ldots b_{n} \hat{a}^{k}\right)=a^{k} b a^{k}$ for $k \geq 0$.

Now, the generating set $W$ of $R$ must contain the word $\hat{a}^{r}$ for some $r \geq 1$. Also, some concatenation of words in $W$ must have the form $\hat{a}^{s} b_{-n} \ldots b_{n} \hat{a}^{t}$, $s, t \geq 0$, or it would be impossible to produce the sequence $\hat{a}^{\infty} b_{-n} \ldots b_{n} \hat{a}^{\infty}$.

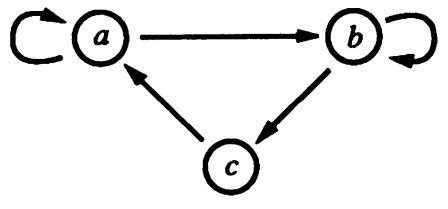

FIGURE 2

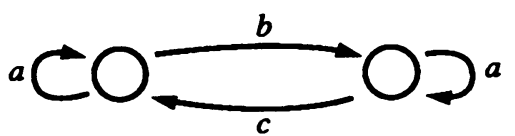

Figure 3 
So, $R$ must contain a point

$$
x=\hat{a}^{\infty} b_{-n} \ldots b_{n} \hat{a}^{m} b_{-n} \ldots b_{n} \hat{a}^{\infty}
$$

with $m \geq 2 n$. But then

$$
\varphi^{-1}(x)=a^{\infty} b a^{m+2 n} b a^{\infty},
$$

which is not a point in $S$.

Note added in proof. Renewal systems are called finitely generated systems by A. Restivo [R], who shows it is decidable when a sofic shift is itself a renewal system.

\section{REFERENCES}

[BMT] M. Boyle, B. Marcus, and P. Trow, Resolving maps and the dimension group for shifts of finite type, Mem. Amer. Math. Soc., no. 377, 1987, pp. 1-146.

[GLS] J. Goldberger, D. Lind, and M. Smorodinsky, The entropies of renewal systems, preprint, 1989.

[M] B. Marcus, Sofic systems and encoding data, IEEE Transactions and Information Theory 31 (1985), 366-377.

[R] A. Restivo, Finitely generated sofic systems, Theoretical Comp. Sci. 65 (1989), 265-270.

[W] B. Weiss, Subshifts of finite type and sofic systems, Monatsh. Math. 77 (1973), 462-474.

University of South Alabama, Department of Mathematics and Statistics, Mobile, Alabama, 36688

E-mail address: f0dm@usouthal.bitnet 